\title{
Mode analysis in optics through fractional transforms
}

\author{
Tatiana Alieva and Martin J. Bastiaans \\ Faculteit Elektrotechniek, Technische Universiteit Eindhoven, Postbus 513, 5600 MB Eindhoven, Netherlands
}

Received May 19, 1999

The relationship between the mode content and the fractional Fourier and fractional Hankel transforms of a function is established. It is shown that the Laguerre-Gauss spectrum of a rotationally symmetric wave front can be determined from the wave front's fractional Hankel transforms taken at the optical axis. (C) 1999 Optical Society of America

OCIS codes: $070.0070,030.4070$.

The Fourier transform, which gives information about the harmonic content of a wave front, is one of the most important tools in wave optics. In certain applications, however, for example, in the analysis of laser cavity modes, knowledge of the mode content is even more important. Recently different kinds of fractional integral transform, which are closely related to the Fourier transform, were introduced in optical signal processing. ${ }^{1,2}$ These transforms describe in the paraxial approximation of the scalar diffraction theory the evolution of the complex field amplitude during wave propagation through quadratic refractive-index media such as lenses, fibers, and mirrors. Here we consider first-order optical systems that are performing a fractional Fourier transform (FT) or a fractional Hankel transform (HT) of the input complex field amplitude $f(\mathbf{x})$. A typical optical setup ${ }^{3}$ contains a thin lens with focal length $f$, situated at the same distance $d=2 f \sin ^{2}(\alpha / 2)$ from the input and the output plane, which are transverse to the optical axis. By applying cylindrical lenses with focal lengths $f_{1}$ and $f_{2}$, one gets at the output plane the fractional FT at the angle $\alpha=\left(\alpha_{1}, \alpha_{2}\right)$ of the input complex field amplitude. In the case of rotational symmetry of the input wave front, $f(\mathbf{x})=f(x)$, a spherical lens performs a fractional $\mathrm{HT}$ at the angle $\alpha$ of the input complex field amplitude. The existence of inverse transforms allows us to determine the input wave front from knowledge of the complex field amplitude at any plane transverse to the optical axis of the fractional FT or HT systems. The input complex field amplitude can also be determined from its Radon-Wigner transform,${ }^{5}$ which corresponds to the intensity distribution at the transverse planes of the fractional FT and the fractional HT systems, taken in the regions $\alpha \in[0, \pi]$ and $\alpha \in[0, \pi / 2]$, respectively. In this case the parameter $\alpha$ as well as the coordinate $\mathbf{u}$ of the transform is considered a variable.

Here we consider the problem of reconstructing an optical signal from its fractional FT or HT, where the coordinate $\mathbf{u}$ at the plane that is transverse to the propagation axis is fixed and the angle $\alpha$ is considered a variable. This problem corresponds to the input complex amplitude characterization from knowledge of the complex field amplitude at a line parallel to the optical axis of the corresponding system. We show how information about the mode content of the input optical signal can be recovered from these measurements.

First we propose a method for the determination of the Hermite-Gauss mode spectra from the fractional FT of the investigated field for all possible combinations of the parameters $\alpha_{1}, \alpha_{2} \in[0,2 \pi]$.

The two-dimensional fractional FT $F\left(u_{1}, u_{2}, \alpha_{1}, \alpha_{2}\right)$ at angles $\alpha_{1}$ and $\alpha_{2}$ of a function $f(\mathbf{x})$ is defined as

$$
\begin{aligned}
F\left(u_{1}, u_{2}, \alpha_{1}, \alpha_{2}\right)= & R^{\alpha_{1}, \alpha_{2}}[f(\mathbf{x})](\mathbf{u}) \\
= & \int_{-\infty}^{\infty} f(\mathbf{x}) K_{\alpha_{1}}\left(x_{1}, u_{1}\right) \\
& \times K_{\alpha_{2}}\left(x_{2}, u_{2}\right) \mathrm{d} \mathbf{x}
\end{aligned}
$$

where

$$
\begin{aligned}
K_{\alpha_{j}}\left(x_{j}, u_{j}\right)= & \left(\frac{1-i \cot \alpha_{j}}{2 \pi}\right)^{1 / 2} \\
& \times \exp \left[i \frac{\cos \alpha_{j}\left(x_{j}^{2}+u_{j}^{2}\right)-2 x_{j} u_{j}}{2 \sin \alpha_{j}}\right] .
\end{aligned}
$$

We assume that the fractional FT operator acts independently on the coordinates $x_{1}$ and $x_{2}$. Thus the transverse-plane amplitude $F\left(u_{1}, u_{2}, \alpha_{1}, \alpha_{2}\right)$ at a distance $2 d$, where $d=2 f_{1} \sin ^{2}\left(\alpha_{1} / 2\right)=2 f_{2} \sin ^{2}\left(\alpha_{2} / 2\right)$ and $f_{1}$ and $f_{2}$ are the focal lengths of the cylindrical lenses situated at the distance $d$ from the input plane, is the fractional FT of the input complex field amplitude $f(\mathbf{x})$ (for which $\alpha_{1}=\alpha_{2}=0$ ). We call such an optical system a fractional FT system, and we assume that $\alpha_{1}$ and $\alpha_{2}$ change from 0 to $2 \pi$.

The eigenfunctions of the fractional FT at any angle $\alpha$ are the Hermite-Gauss modes $\Psi_{n}(x)$ defined by

$$
\Psi_{n}(x)=\left(\sqrt{\pi} 2^{n} n !\right)^{-1 / 2} \exp \left(-x^{2} / 2\right) H_{n}(x),
$$

where $H_{n}(x)$ are the Hermite polynomials. Thus

$$
R^{\alpha}\left[\Psi_{n}(x)\right](u)=\exp (-i \alpha n) \Psi_{n}(u),
$$

where $\exp (-i \alpha n)$ is the eigenvalue of the eigenfunction $\Psi_{n}$.

(C) 1999 Optical Society of America 
Since the Hermite-Gauss functions form a complete orthonormal set,

$$
\int_{-\infty}^{\infty} \Psi_{n}(x) \Psi_{m}(x) \mathrm{d} x=\left\{\begin{array}{ll}
0 & n \neq m \\
1 & n=m
\end{array},\right.
$$

a complex field amplitude $f(\mathbf{x})$ can be expanded as

$$
f(\mathbf{x})=\sum_{n=0}^{\infty} \sum_{m=0}^{\infty} f_{n, m} \Psi_{n}\left(x_{1}\right) \Psi_{m}\left(x_{2}\right),
$$

where

$$
f_{n, m}=\int_{-\infty}^{\infty} \int_{-\infty}^{\infty} \Psi_{n}\left(x_{1}\right) \Psi_{m}\left(x_{2}\right) f\left(x_{1}, x_{2}\right) \mathrm{d} \mathrm{x}_{1} \mathrm{~d} x_{2} .
$$

Thus the set of coefficients $\left\{f_{n, m}\right\}$, which we call the Hermite-Gauss spectrum, completely determines the function $f(\mathbf{x})$.

Using Eqs. (1), (4), and (6), we can write the expression for the fractional FT at angles $\alpha_{1}$ and $\alpha_{2}$ of the input wave front $f(\mathbf{x})$ in the form

$$
\begin{aligned}
F\left(u_{1}, u_{2}, \alpha_{1}, \alpha_{2}\right)= & R^{\alpha_{1}, \alpha_{2}}[f(\mathbf{x})](\mathbf{u}) \\
= & \sum_{n=0}^{\infty} \sum_{m=0}^{\infty} f_{n, m} \exp \left[-i\left(\alpha_{1} n+\alpha_{2} m\right)\right] \\
& \times \Psi_{n}\left(u_{1}\right) \Psi_{m}\left(u_{2}\right)
\end{aligned}
$$

Considering $F\left(u_{1}, u_{2}, \alpha_{1}, \alpha_{2}\right)$ as a function of $\alpha_{j}$, it is easy to see that the set of products of the HermiteGauss coefficients $f_{n, m}$ and $\Psi_{n}\left(u_{1}\right)$ and $\Psi_{m}\left(u_{2}\right)$ for fixed coordinates $u_{1}$ and $u_{2}$ constitutes the harmonic-mode content of the periodic function $F\left(u_{1}, u_{2}, \alpha_{1}, \alpha_{2}\right)$. The inverse two-dimensional FT of $F\left(u_{1}, u_{2}, \alpha_{1}, \alpha_{2}\right)$ with respect to the parameters $\alpha_{1}$ and $\alpha_{2}$,

$$
\begin{aligned}
Z(\mathbf{u}, \mathbf{p})= & \int_{0}^{2 \pi} \int_{0}^{2 \pi} R^{\alpha_{1}, \alpha_{2}}[f(\mathbf{x})](\mathbf{u}) \\
& \times \exp \left[i\left(\alpha_{1} p_{1}+\alpha_{2} p_{2}\right)\right] \mathrm{d} \alpha_{1} \mathrm{~d} \alpha_{2},
\end{aligned}
$$

produces the discrete $\mathbf{p}$ function $Z(\mathbf{u}, \mathbf{p})$,

$$
\begin{aligned}
Z(\mathbf{u}, \mathbf{p})= & \sum_{n=0}^{\infty} \sum_{m=0}^{\infty} f_{n, m} \Psi_{n}\left(u_{1}\right) \Psi_{m}\left(u_{2}\right) \\
& \times \delta\left(p_{1}-n\right) \delta\left(p_{2}-m\right),
\end{aligned}
$$

which for a fixed value $\mathbf{u}=\mathbf{u}_{0}$ corresponds to the Hermite-Gauss spectrum $f_{n, m}$ modulated by $\Psi_{n}\left(u_{1,0}\right) \Psi_{m}\left(u_{2,0}\right)$. This implies that $Z(\mathbf{u}, \mathbf{p})$ can be different from zero only at the points $p_{1}=n$ and $p_{2}=m$ :

$$
Z\left(\mathbf{u}_{0}, n, m\right)=f_{n, m} \Psi_{n}\left(u_{1,0}\right) \Psi_{m}\left(u_{2,0}\right) .
$$

We conclude that the complex field amplitude at any transverse plane can be determined from knowledge of its values along the lines parallel to the optical axis of the fractional FT system.

Note that for a given value $\mathbf{u}_{0}$ the Hermite-Gauss function of certain indices $n_{j}$ and $m_{j}$ can be equal to zero, in which case one has to choose a different value $\mathbf{u}$ for recovering the Hermite-Gauss spectrum component $f_{n_{j}, m_{j}}$. For $\mathbf{u}_{0}=0$, for instance, all odd-order coefficients in Eq. (10) are zero, since $\Psi_{2 n+1}(0)=0$ for $n=0,1, \ldots$, whereas the even-order coefficients read as

$$
Z(0,2 n, 2 m)=f_{2 n, 2 m} \Psi_{2 n}(0) \Psi_{2 m}(0) .
$$

In this case we can determine only the spectrum of the even-order Hermite-Gauss modes, which is sufficient only for an even function $f(\mathbf{x})=f(-\mathbf{x})$. Note that the modulating coefficients are given by

$$
\Psi_{2 n}(0)=\frac{(-1)^{n} \sqrt{(2 n) !}}{2^{n} \pi^{1 / 4} n ! n} .
$$

To determine all spectral components we then have to choose a different line $u=u_{0} \neq 0$ that is parallel to the optical axis of the fractional FT system.

The simplest results can be obtained in the case of rotationally symmetric complex field amplitudes $f(\mathbf{x})=$ $f(x)$, where $x=\sqrt{x_{1}^{2}+x_{2}^{2}}$. The two-dimensional fractional FT at identical angles for the two orthogonal coordinates of the rotationally symmetric function reduces to a fractional $\mathrm{HT}$ of zero order.

The fractional HT of zero order at an angle $\alpha$ of a function $f(x)$ is defined $\mathrm{as}^{2}$

$$
F(u, \alpha)=R^{\alpha}[f(x)](u)=\int_{0}^{\infty} f(x) H_{\alpha}(x, u) x \mathrm{~d} x,
$$

where

$$
\begin{aligned}
H_{\alpha}(x, u)= & (1-i \cot \alpha) J_{0}(x u / \sin \alpha) \\
& \times \exp \left[i \cot \alpha\left(x^{2}+u^{2}\right) / 2\right]
\end{aligned}
$$

and $J_{0}(x)$ is a Bessel function of order zero. It is easy to see from Eq. (14) that $F(u, \alpha)$ is periodic in parameter $\alpha$, with period $\pi$.

The eigenfunctions of the fractional HT at any angle $\alpha$ are the Laguerre-Gauss modes defined by

$$
\Phi_{n}(x)=\sqrt{2} L_{n}\left(x^{2}\right) \exp \left(-x^{2} / 2\right),
$$

where $L_{n}(x)$ are the Laguerre polynomials. Thus

$$
R^{\alpha}\left[\Phi_{n}(x)\right](u)=\exp (-2 i \alpha n) \Phi_{n}(u),
$$

where $\exp (-2 i \alpha n)$ is the eigenvalue of the eigenfunction $\Phi_{n}$.

Since the Laguerre-Gauss functions $\Phi_{n}$, such as the Hermite-Gauss functions $\Psi_{n}$ [see Eq. (5)], form a complete orthonormal set,

$$
\int_{0}^{\infty} \Phi_{n}(x) \Phi_{m}(x) x \mathrm{~d} x= \begin{cases}0 & n \neq m \\ 1 & n=m\end{cases}
$$

a rotationally symmetric complex field amplitude $f(x)$ can be expanded as

$$
f(x)=\sum_{n=0}^{\infty} f_{n} \Phi_{n}(x),
$$


where

$$
S(0, n)=f_{n}
$$

$$
f_{n}=\int_{0}^{\infty} \Phi_{n}(x) f(x) x \mathrm{~d} x .
$$

Thus the set of coefficients $\left\{f_{n}\right\}$, which we call the Laguerre-Gauss mode spectrum, completely determines the function $f(x)$.

Using Eqs. (14), (17), and (19), we can write the expression for the fractional HT at angle $\alpha$ of the input wave front in the form

$$
F(u, \alpha)=\sum_{n=0}^{\infty} f_{n} \exp (-2 i \alpha n) \Phi_{n}(u) .
$$

Considering $F(u, \alpha)$ as a function of $\alpha$, it is easy to see that the set of products of the LaguerreGauss coefficients $f_{n}$ and $\Phi_{n}(u)$ for fixed $u$ constitutes the harmonic-mode content of the periodic function $F(u, \alpha)$. The inverse FT of $F(u, \alpha)$ with respect to the parameter $\alpha$,

$$
S(u, p)=\int_{0}^{\pi} F(u, \alpha) \exp (i 2 \alpha p) \mathrm{d} \alpha,
$$

produces the discrete $p$ function $S(u, p)$,

$$
S(u, p)=\sum_{n=0}^{\infty} f_{n} \Phi_{n}(u) \delta(p-n),
$$

which for a fixed value $u=u_{0}$ corresponds to the Laguerre-Gauss spectrum $f_{n}$ modulated by $\Phi_{n}\left(u_{0}\right)$. This implies that $S(u, p)$ can be different from 0 only at the points $p=n$ :

$$
S\left(u_{0}, n\right)=f_{n} \Phi_{n}\left(u_{0}\right) .
$$

Since $\Phi_{n}(0)=1, S(0, n)$ reduces to the LaguerreGauss spectrum

Equation (24) corresponds to knowledge of the complex field amplitude in the fractional HT system on the optical axis $F(0, \alpha)$ and application of an inverse FT.

So we conclude that a rotationally symmetric input field can be completely determined from knowledge of the complex field amplitude along the optical axis in the fractional HT system for $\alpha \in[0, \pi]$.

If only the average of $F(u, \alpha)$ on the coordinate $u$ can be measured,

$$
\langle F(u, \alpha)\rangle=\int_{0}^{\rho} F(u, \alpha) \mathrm{d} u
$$

and $\langle F(u, \alpha)\rangle \neq 0$, then the Laguerre-Gauss spectrum can be determined as

$$
f_{n}=\langle S(u, n)\rangle /\langle F(u, \alpha)\rangle .
$$

We conclude that the fractional FT and HT, when they are treated as functions of the angle $\alpha$, with the coordinate considered a fixed parameter, allow us to recover the input function and determine its mode content.

\section{References}

1. D. Mendlovic and H. M. Ozaktas, J. Opt. Soc. Am. A 10, 1875 (1993).

2. L. Yu, Y. Ku, X. Zheng, M. C. Huang, M. Chen, W. Huang, and Z. Zhu, Opt. Lett. 23, 1158 (1998).

3. A. W. Lohmann, J. Opt. Soc. Am. A 10, 2181 (1993).

4. A. Sahin, H. M. Ozaktas, and D. Mendlovic, Appl. Opt. 37, 2130 (1998).

5. M. G. Raymer, M. Beck, and D. F. McAlister, Phys. Rev. Lett. 72, 1137 (1994). 